\title{
MICROFABRICATION OF FREESTANDING METAL STRUCTURES RELEASED FROM
}

\author{
GRAPHITE SUBSTRATES \\ Olga V. Makarova ${ }^{*}$, Cha-Mei Tang ${ }^{*}$, Derrick C. Mancini* ${ }^{* *}$, Nicolaie Moldovan ${ }^{* *}$, \\ Ralu Divan ${ }^{* *}$, David G. Ryding ${ }^{* *}$, Richard H. Lee" \\ ${ }^{*}$ Creatv MicroTech, Inc. Potomac, MD 20854, \\ *** Advanced Photon Source, Argonne National Laboratory, Argonne, IL 60439
}

\begin{abstract}
A sacrificial layer is usually used to release electroformed microstructures. Because of the chemistry applied to the sacrificial layer, only a limited number of metals can be used for electroforming. A novel method to fabricate freestanding electroformed copper structures is presented. A graphite substrate allows the release of the metal part, by abrasive removal of the graphite after electroforming. Results on fabrication of high-aspect-ratio freestanding copper grids are presented; these can be used as $\mathrm{x}$-ray collimator in medical imaging to reduce scattered radiation. This process has potential application to the fabrication of injection molds and microparts on pick-and-place carriers for microelectromechanical systems (MEMS).
\end{abstract}

\section{INTRODUCTION}

The fabrication of high-aspect-ratio microstructures (HARMS) using deep x-ray lithography (DXRL) and electroforming requires that the substrate provides good resist adhesion before and after exposure, that the substrate has a conductivity sufficient for the subsequent electroforming process, and that the metal structure can be released from the substrate after electroforming. Metal-coated silicon wafers are usually used as a primary substrate. The high conductivity of the metal layer makes the wafer suitable as an electroplating base, but the secondary radiation generated during the higher energy exposure of DXRL can lead to adhesion failure [1]. Adhesion buffer layers have been used to reduce adhesion failure [2], but they complicate processing and can be difficult to remove. A sacrificial layer is usually used to release the electroformed metal part from the substrate. This method limits the number of metals that can be used for electroforming because of the chemical requirements on the sacrificial layer. For example, fabrication of a freestanding copper microstructure is a problem, because the wet etch that removes the release layer may also attack the copper structure.

Recently, thin conductive carbon films have been used with some success as a plating base [3]. Rigid graphite has been used as a substrate for the fabrication of masks for DXRL [4], generally with a plating base prepared by precision resurfacing and metal coating. Properties of rigid graphite, such as rigidity, low cost, and good thermal and electrical conductivity, suggest that bare graphite would be a suitable substrate and plating base for fabrication of
HARMS using DXRL and electroforming. A bare graphite substrate may also be preferred over silicon wafers, which are traditionally used, because carbon has a smaller atomic number than both silicon and metal coatings $(\mathrm{Au}, \mathrm{Ti}, \mathrm{Cu})$, and thus generates less secondary radiation during $\mathrm{X}$-ray exposure.

Mammography is an application that requires a freestanding, very high-aspect-ratio, antiscatter grid for x-ray energy primarily in the range of $17-35 \mathrm{keV}$. Copper is a preferred electroplating material because of adequate absorption of scattered x-ray, low toxicity electroforming bath, and low cost.

\section{EXPERIMENTAL DETAILS}

Commercially available rigid graphite sheets (Goodfellow Corp.), ranging in thickness from 0.25 $\mathrm{mm}$ to $1 \mathrm{~mm}$, were used as the primary substrate and as the plating base for the microfabrication. PMMA sheets 1 or more $\mathrm{mm}$ thick were used as a resist layer. The graphite substrate was cleaned with acetone and spin-coated with a 5- $\mu$ m-thick PMMA resist layer $\left(\mathrm{M}_{\mathrm{w}}=2200 \mathrm{~K}, 10 \%\right.$ in anizole $)$; after it had dried at room temperature for several days, a 1mm-thick PMMA sheet was solvent-bonded on top of the PMMA layer using methylmethacrylate (MMA) [5]. After drying MMA for a day at room temperature, the substrate could then be exposed. Hard x-ray exposures were performed at beamline 2BM (bending magnet) [6] at the Advanced Photon Source. The beam size was $100 \times 5 \mathrm{~mm}^{2}$, and the photon energy was $10-20 \mathrm{keV}$, after passing through a $1 \mathrm{~mm}$ carbon filter and reflecting from a $0.15^{\circ}$ grazing-incidence chromium mirror. X-ray masks used for patterning were fabricated by conformal 
mask technology [7] on 250- $\mu$ m-thick silicon wafers, and the thickness of the gold absorber was 45-60 $\mu \mathrm{m}$. The exposed PMMA was developed using the G-G developing system [8]. Copper electroforming was performed using a copper sulfate plating process from LeaRonal TechSpec. After electroforming, the copper microstructures along with the PMMA mold were released from the plating base by abrasive removal of graphite. Both sides of the copper microstructure were polished using aluminum oxide pads. Then the PMMA mold was dissolved in acetone, resulting in the finished freestanding metal part. The schematic of the fabrication method is shown in Fig. 1.

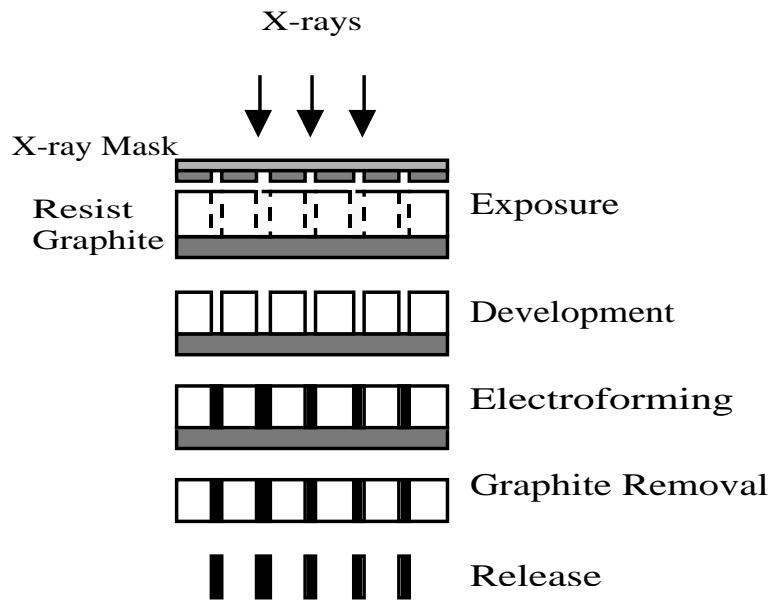

Figure 1. Process steps for manufacturing freestanding metal microstructures using DXRL and electroforming on a graphite substrate.

\section{RESULTS AND DISCUSSION}

PMMA structures patterned on the bare graphite substrate exhibited significantly better adhesion compared with metal-coated silicon or graphite due to the greater microroughness and porosity of the bare graphite surface [9]. Rigid graphite is $80 \%$ dense, with mean pore size of $0.8 \mu \mathrm{m}$. Spin coating of graphite with about 5- $\mu$ m-thick PMMA and resist-drying at room temperature were found to be optimal, while a thinner PMMA layer led to adhesion failure, due to significant absorption of resist by the graphite.

The conductivity of graphite is sufficient to perform electroplating directly without the need for a metal plating base layer. Graphite turns out to be an excellent plating base for copper electroforming using acid sulfate electrolyte, and gold electroforming using sulfite electrolyte.
Although copper deposition on the graphite surface usually starts well, for very high-aspect-ratio structures it was necessary to apply electrochemical activation of the carbon surface (reverse current) to initiate copper electroplating.

In many cases, the metal part must be separated from the plating base to provide a freestanding metallic structure. The graphite substrate can be easily removed by abrasive methods once electroforming is complete. After graphite removing, the PMMA mold can be dissolved, resulting in a freestanding metal part.

By using rigid graphite as the substrate for sample preparation, freestanding copper antiscatter grids can be fabricated for mammography [10]. These grids can significantly improve the image quality of the mammography due to its high transparency to primary radiation and its ability to absorb scattered radiation from all directions. A scanning electron microscope (SEM) image of a 1.5-mm-thick freestanding copper grid for mammography with an aspect ratio over 60 is shown in Fig. 2.

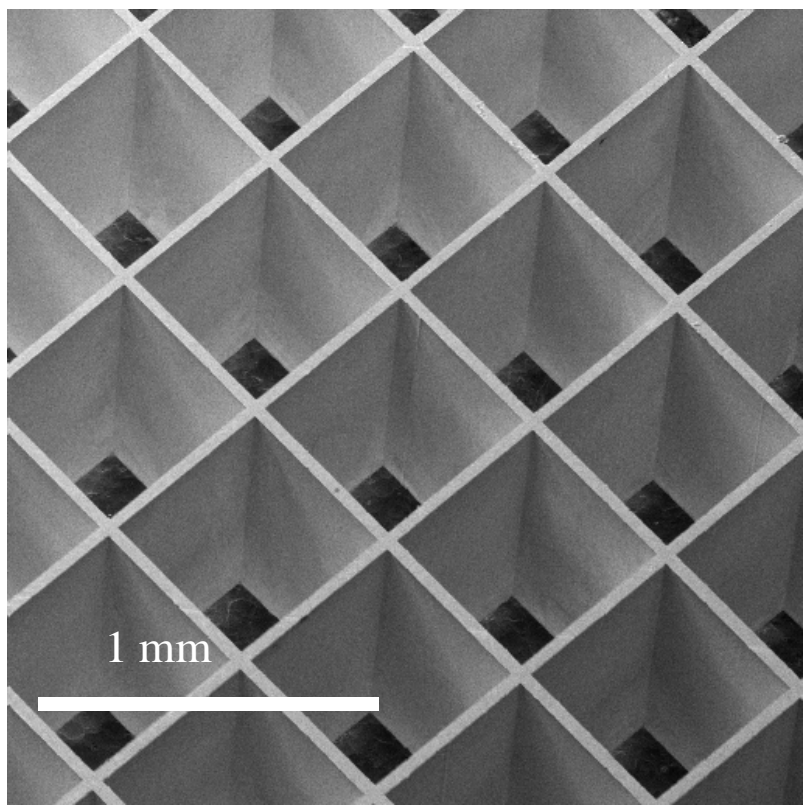

Figure 2. A scanning electron micrograph of a $1.5-\mathrm{mm}-$ thick freestanding copper grid with $25-\mu \mathrm{m}$-thick cell walls and $550 \mu \mathrm{m}$ period.

In Fig. 3, two other processes are shown that demonstrate the application to MEMS fabrication. There is potential application of this process to the 
fabrication of both injection molds and microparts on pick-and-place carriers for microelectromechanical systems (MEMS). Micromolds can be fabricated by first electroforming in an appropriate metal such as nickel and then overplating additional nickel to form the base of the mold (Fig. 3a). After graphite and PMMA are removed, the remaining metal part can be used for embossing or injection molding thermoplastics or slurries to produced polymer or ceramic microparts for assembly into MEMS. For the production of many discrete microparts in electroformed metals for later assembly into MEMS, this batch of microparts must be released, yet temporarily held to be later individually picked from the batch and placed into MEMS. This pick-and-place assembly can be facilitated by attaching a carrier to the array of electroformed parts while still attached to the graphite. The carrier may be attached by means of temporary adhesive or magnetic forces in the case of ferrous and ferromagnetic parts, such as nickel and permalloy. After graphite and PMMA are removed, the individual parts may be picked off of the carrier and placed into MEMS manually or with automated equipment.

\section{Electroforming}

a) Overplate

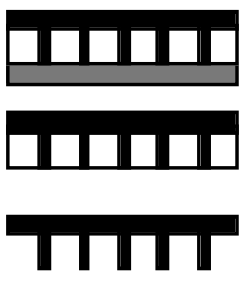

b) Attach

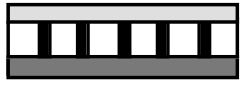

Graphite Removal

\section{Resist}

Removal
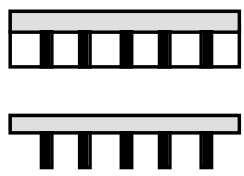

Figure 3. Process steps (after the metal electroforming in Fig. 1) for the fabrication of (a) an injection mold for MEMS manufacture and (b) arrays of small MEMS components for subsequent pick-and-place assembly.

\section{CONCLUSION}

We have been successful in the development of a process that uses a graphite substrate to fabricate freestanding metal microstructures. This process has been applied to the production of freestanding copper antiscatter grids for mammography. It has potential in the production of micromolds for injection mold fabrication of MEMS, and for fabrication of arrays of microparts on pick-and-place carriers for assembly into MEMS.

\section{ACKNOWLEDGMENTS}

The work is supported by NIH SBIR Phase II Grants: 2 R44 CA76752-02 and 5 R44 CA76752-03, and by U.S. Department of Energy, Office of Sciences, under Contract No. W-31-109-ENG-38.

\section{REFERENCES}

[1] F. J. Pantenburg, J. Chlebek, A. El-Kholi, H.-L. Huber, J. Mohr, H. K. Oertel, J. Schulz, "Adhesion problems in deep-etch X-ray lithography caused by fluorescence radiation from the plating base", Microelectronic Eng. Vol. 23 (1994) 223-226.

[2] F. DeCarlo, J. J. Song, D. C. Mancini, "Enhanced Adhesion Buffer Layer for Deep X-Ray Lithography Using Hard X-Rays", J. Vac. Sci. Technol. B, Vol. 16 (1998) 3539-3542.

[3] A. El-Kholi, K. Bade, J. Mohr, F. J. Pantenburg, X.M. Tang, "Alternative resist adhesion and electroplating layers for LIGA process", Microsystems Technol., Vol. 6 (2000) 161-164.

[4] P. Coane, F. DeCarlo, Y. Desta, R. Giasolli, J. Göttert, D. C. Mancini, "Graphite-Based X-ray Masks for Deep and Ultra-deep X-ray Lithography", J. Vac. Sci. Technol. B, Vol. 16 (1998) 3618-3624.

[5] H. Guckel, T. R. Christenson, K. Skrobis, "Formation of microstructures using a preformed photoresist", U.S. Patent 5,378,583, 1995.

[6] B. Lai, D. C. Mancini, W. Yun, and E. Gluskin, "Beamline and station for deep x-ray lithography at the Advanced Photon Source", Proc. SPIE Vol. 2880 (1996) 171-6.

[7] V. White, C. Herdey, D. D. Denton, J. Song, "X-ray fabrication of nonorthogonal structures using 'surface' masks", J. Vac. Sci. Technol. B Vol. 15 (1997) 2514-6.

[8] C. H. Malek and S. Yajamanyam, Evaluation of alternative development process for high-aspectratio poly(methylmethacrylate) microstructures in deep x-ray lithography", J. Vac. Sci. Technol. B Vol. 18 (2000) 3354.

[9] D. C. Mancini, N. Moldovan, O. V. Makarova, A. G.Peele, T. H. K. Irving, "Use of graphite substrates for deep x-ray lithography", Microsystem Technol. (2001), submitted.

[10] O. V. Makarova, C.-M. Tang, D. C. Mancini, N. Moldovan, R. Divan, D. G. Ryding, R. H. Lee "Development of freestanding copper anti-scatter grid using deep x-ray lithography", Microsystem Technol. (2001), submitted. 